EXTENDED REPORT

\title{
Validation of the preliminary criteria for the classification of catastrophic antiphospholipid syndrome
}

\author{
R Cervera, J Font, J A Gómez-Puerta, G Espinosa, M Cucho, S Bucciarelli, M Ramos- \\ Casals, M Ingelmo, J-C Piette, Y Shoenfeld, R A Asherson for the Catastrophic \\ Antiphospholipid Syndrome Registry Project Group*
}

See end of article for authors' affiliations

Correspondence to: Dr Ricard Cervera, Servei de Malalties Autoimmunes, Hospital Clínic, Villarroel 170, 08036-Barcelona, Catalonia, Spain; rcervera@clinic.ub.es

*The members of the Catastrophic

Antiphospholipid Syndrome Registry Project Group are listed in the appendix

Accepted 30 January 2005 Published Online First 11 February 2005
Ann Rheum Dis 2005;64:1205-1209. doi: 10.1136/ard.2004.025759

Objective: To describe the characteristics of patients with catastrophic antiphospholipid syndrome (APS) included in the International Registry of patients with this condition (CAPS registry) and to analyse the value of the recently proposed preliminary criteria for the classification of catastrophic APS.

Methods: A review of the first 220 patients included in the website based CAPS registry was undertaken and the preliminary criteria for their classification were tested; 175 unselected patients with systemic lupus erythematosus or APS, or both, acted as controls.

Results: The mean age of the patients was 38 (14) years (range 7 to 74), with a female preponderance (F/ $M, 153 / 67)$. The main clinical manifestations included renal involvement in 154 (70\%), pulmonary in 146 (66\%), cerebral in 133 (60\%), cardiac in 115 (52\%), and cutaneous in 104 (47\%); 114 patients (52\%) recovered after the catastrophic APS event (mortality 48\%). Patients who received the combination of anticoagulation plus steroids plus plasma exchange or intravenous immunoglobulins had the best survival rate $(63 \%, p=0.09)$. Sufficient data could be analysed for application of the classification criteria in 176 patients. According to the preliminary criteria, 89 patients (51\%) could be classified as having "definite" and $70(40 \%)$ as having "probable" catastrophic APS, thus given a sensitivity of $90.3 \%$ with a specificity of $99.4 \%$. Positive and negative predictive values were $99.4 \%$ and $91.1 \%$, respectively.

Conclusions: The preliminary criteria for the classification of catastrophic APS and the CAPS registry are useful tools for epidemiological studies.
I n 1992, the "catastrophic" antiphospholipid syndrome (APS) was first defined as a potential life threatening variant of the APS which is characterised by multiple small vessel thrombosis that can lead to multiorgan failure. ${ }^{1}$ Fortunately, this is an unusual form of presentation that represents fewer than $1 \%$ of the APS cases. ${ }^{2}$ The recurrence rate is low with a stable clinical course if these patients are treated with adequate anticoagulation. ${ }^{3}$ Owing to the rarity of its presentation, an international registry of patients (the CAPS registry) was created in 2000 supported by the European Forum on Antiphospholipid Antibodies (aPL).

The heterogeneity of the different clinical forms of presentation led to the need to develop consensus criteria for the classification of this condition. In 2002, a precongress workshop at the Tenth International Congress on aPL held in Taormina, Italy, allowed the establishment of the preliminary criteria for the classification of catastrophic APS that were published recently. ${ }^{4}$ The objectives of the present study were to describe the characteristics of the patients with catastrophic APS included in the CAPS registry and to analyse the value of the preliminary criteria for their classification using the data from this registry.

\section{METHODS}

We reviewed the 220 patients who were included in the website based international registry of patients with catastrophic APS (CAPS registry) at 1 October 2003, and tested the recently proposed preliminary criteria for the classification of catastrophic APS ${ }^{4}$ in those patients whose clinical data were sufficient for application of the criteria. The CAPS registry compiles all the published reports as well as newly diagnosed cases of catastrophic APS from all over the world. The diagnoses and data have been submitted by a wide variety of interested clinicians, but efforts were made in most cases to contact these clinicians and verify the accuracy of the data sent in. The basis for submitting patient data was clinical judgement, as no classification criteria were published until 2003. The different variables of the database are detailed at http://www.med.ub.es/MIMMUN/FORUM/ CAPS.HTM.

Additionally, we analysed 175 unselected patients from our clinics as controls: 100 with systemic lupus erythematosus (SLE), classified according to the American College of Rheumatology (ACR) revised criteria ${ }^{5}$-all with positive aPL and 65 with associated APS-and 75 with primary APS, fulfilling the preliminary criteria for the classification of definite APS.

\section{Data analysis}

Conventional Fisher's exact test was used for analysing qualitative differences. When several independent variables appeared to have statistical significance in the univariate analysis, a logistic regression test was carried out for multivariate analysis in order to rule out possible confounding variables. In this case, only those variables showing statistical significance in the multivariate analysis were considered to be significant study results. The sensitivity,

Abbreviations: aCL, anticardiolipin antibodies; $\mathrm{ACR}$, American College of Rheumatology; APL, antiphospholipid antibodies; APS, antiphospholipid syndrome; CAPS, catastrophic antiphospholipid syndrome; HELLP, haemolysis, elevated liver enzymes, and low platelet count syndrome; SLE, systemic lupus erythematosus 
Table 1 Previous antiphospholipid syndrome manifestations of the patients from the CAPS registry

\begin{tabular}{lc}
\hline Manifestation & $\mathbf{n}(\%)$ \\
\hline Deep vein thrombosis & $44(20)$ \\
Fetal loss & $31(20)^{*}$ \\
Thrombocytopenia & $29(13)$ \\
Cerebrovascular accident & $20(9)$ \\
Skin ulcers & $19(9)$ \\
Pulmonary embolism & $18(8)$ \\
Livedo reticularis & $17(8)$ \\
Peripheral artery thrombosis & $10(5)$ \\
Myocardial infarction & $9(4)$ \\
Haemolytic anaemia & $7(3)$ \\
Seizures & $7(3)$ \\
Digital ischaemia & $7(3)$ \\
Valve lesions & $5(2)$ \\
No previous APS manifestations & $104(47)$ \\
\hline *Percentage relates to the female patient population. \\
APS, antiphospholipid syndrome.
\end{tabular}

specificity, and predictive values of the preliminary criteria for the classification of catastrophic APS were determined according to Galen and Gambino. ${ }^{7}$

\section{RESULTS}

\section{General characteristics of patients with catastrophic APS}

The mean (SD) age was 38 (14) years (range 7 to 74 ) with a female preponderance (F/M, 153/67); 106 (48\%) suffered from primary APS, 88 (40\%) from SLE, 11 (5\%) from lupuslike syndrome, four (2\%) from rheumatoid arthritis, four (2\%) from systemic sclerosis, and the remaining seven (3\%) from other autoimmune disorders (relapsing polychondritis, ulcerative colitis, Crohn's disease, dermatomyositis, and Behçet's disease).

\section{Clinical presentation and precipitating factors}

Fifty three per cent of the patients had previous APS manifestations (table 1). The main previous manifestations were deep vein thrombosis in $44(20 \%)$, fetal loss (abortions or fetal deaths) in 31 female patients $(20 \%)$, thrombocytopenia in $29(13 \%)$, cerebrovascular accidents in 20 (9\%), skin ulcers in $19(9 \%)$, pulmonary embolism in $18(8 \%)$, and livedo reticularis in $17(8 \%)$.

In $58 \%$ of the patients, an identifiable precipitating factor was detected, including infections $(20 \%)$, surgical procedures (biopsies, dental extractions, invasive procedures, transplantation) (14\%), neoplasms (9\%), anticoagulation withdrawal or low international normalised ratio (INR) $(7 \%)$, obstetric complications (5\%), lupus flares $(4 \%)$, and the use of oral contraceptives $(3 \%)$. Twelve patients had two identifiable precipitating factors and in one case three triggering factors were found (anticoagulant withdrawal and surgical resection for a neoplastic process).

The majority of patients presented with multiple organ involvement at the time of catastrophic APS. The combination of pulmonary, cardiac, and renal involvement was most commonly seen. Table 2 shows the thrombotic manifestations described in these patients. However, as some types of organ involvement were detected at necropsy or during surgical procedures and other types can only be scored as present if the clinician actively looks for them, the percentages given may be an underestimate.

\section{Laboratory findings}

The following antibodies were detected: IgG anticardiolipin antibodies (aCL) in 176 of 210 patients (84\%) (in 68 cases in high titres, defined according to the APS classification
Table 2 Clinical manifestations at the time of presentation with catastrophic antiphospholipid syndrome in patients from the CAPS registry

\begin{tabular}{|c|c|}
\hline Feature & n (\%) \\
\hline Peripheral thrombosis & 74 (34) \\
\hline Deep vein thrombosis & $50(23)$ \\
\hline Femoral artery & $8(4)$ \\
\hline Radial artery & $4(2)$ \\
\hline Other arteries & $19(9)$ \\
\hline Cerebral & $133(60)$ \\
\hline Infarcts & $97(44)$ \\
\hline Encephalopathy & $17(8)$ \\
\hline Seizures & $13(6)$ \\
\hline Microthrombosis & $10(5)$ \\
\hline Venous cerebral thrombosis & $5(2)$ \\
\hline Coma & $4(2)$ \\
\hline Transient ischaemic attack & $2(1)$ \\
\hline Cardiac & $115(52)$ \\
\hline Valve lesion & $56(26)$ \\
\hline Myocardial infarction & $50(23)$ \\
\hline Heart failure & $22(10)$ \\
\hline Microthrombosis & $10(5)$ \\
\hline Mural thrombi & $9(4)$ \\
\hline Pulmonary & $146(66)$ \\
\hline Acute RDS & 74 (34) \\
\hline Pulmonary embolism & $54(24)$ \\
\hline Pulmonary haemorrhage & $16(7)$ \\
\hline Microthrombosis & $10(5)$ \\
\hline Pulmonary oedema & $7(3)$ \\
\hline Infarction & $6(3)$ \\
\hline Abdominal & $189(86)$ \\
\hline Renal & $154(70)$ \\
\hline Hepatic & $62(28)$ \\
\hline Splenic & 41 (19) \\
\hline Adrenal & 33 (15) \\
\hline Intestinal & 27 (12) \\
\hline Mesenteric & $23(11)$ \\
\hline Pancreas & $21(10)$ \\
\hline Portal vein thrombosis & $7(3)$ \\
\hline Inferior cava thrombosis & $7(3)$ \\
\hline Gallbladder thrombosis & $6(3)$ \\
\hline Skin & $104(47)$ \\
\hline Livedo reticularis & $62(28)$ \\
\hline Skin ulcers & 30 (14) \\
\hline Digital ischaemia & 21 (10) \\
\hline Purpura & $12(6)$ \\
\hline Necrosis & $7(3)$ \\
\hline Microthrombosis & $7(3)$ \\
\hline Splinter haemorrhages & $5(2)$ \\
\hline Other manifestations & $56(25)$ \\
\hline Retinal artery thrombosis & $11(5)$ \\
\hline Bone marrow necrosis & 7 (3) \\
\hline Uterus & $7(3)$ \\
\hline Neuropathy & $7(3)$ \\
\hline Testicles & $4(2)$ \\
\hline Retinal vein thrombosis & $4(2)$ \\
\hline Thyroid thrombosis & $3(1)$ \\
\hline Avascular necrosis & $4(2)$ \\
\hline Others & $8(4)$ \\
\hline
\end{tabular}

criteria) $)^{6}$; IgM aCL in 80 of 197 (41\%) (in 20 cases in high titres and in 73 cases in association with IgG aCL); lupus anticoagulant in 154 of 203 (76\%); antinuclear antibodies in 113 of $183(62 \%)$; anti-double-stranded DNA antibodies in 60 of $168(36 \%)$; and antibodies to extractable nuclear antigens in 29 of $128(23 \%)$. Thrombocytopenia was found in 129 of 204 patients $(63 \%)$, haemolytic anaemia in 63 of $196(32 \%)$, disseminated intravascular coagulation (DIC) in 39 of 187 $(21 \%)$, and schistocytes in peripheral smear in 21 of 174 (12\%). 


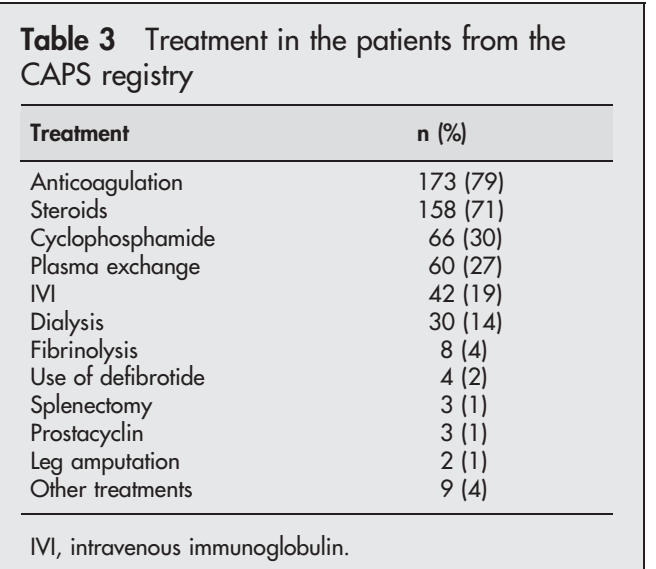

\section{Treatment and outcome}

The different treatments used in patients with catastrophic APS are summarised in table 3. One hundred and fourteen patients $(52 \%)$ recovered after the catastrophic APS event, while the remaining 106 (48\%) died. Some clinical manifestations were related to a worst prognosis (death), such as renal involvement $(\mathrm{p}=0.004$; odds ratio $(\mathrm{OR})=2.4 \quad(95 \%$ confidence interval (CI), 1.21 to 4.76$)$ ), splenic involvement $(\mathrm{p}=0.004 ; \mathrm{OR}=2.63$ (1.2 to 5.84)), pulmonary involvement $(p=0.006 ; \quad O R=1.97 \quad(1.06$ to 3.69$))$, SLE diagnosis $(\mathrm{p}=0.009 ; \mathrm{OR}=1.9(1.01$ to 3.56$))$, and adrenal involvement $(\mathrm{p}=0.05 ; \mathrm{OR}=2.64(1.1$ to 6.44$))$. Those patients who received the combination of anticoagulation plus steroids plus plasma exchange or intravenous immunoglobulins had the best survival rate $(63 \%, \mathrm{p}=0.09)$.

\section{Analysis of the preliminary criteria for classification of the catastrophic APS}

Preliminary criteria for the classification of catastrophic APS are shown in table 4 . From the 220 patients included in the CAPS registry, we were able to analyse enough data for criteria application in 176 patients. In the remaining cases, data on the time of development of manifestations could not be obtained accurately. One hundred and seventy one patients $(97 \%)$ fulfilled the first criterion, $175(99 \%)$ the second, $133(76 \%)$ the third, and $159(90 \%)$ the fourth. With respect to the number of criteria fulfilled, 89 patients $(51 \%)$ fulfilled all four criteria, 74 (42\%) fulfilled three, 11 (6\%) fulfilled two, and two (1\%) fulfilled only one criterion. According to definition of the preliminary classification criteria, $89(51 \%)$ of the previously compiled catastrophic APS patients from the CAPS registry could be classified as having "definite" catastrophic APS (they fulfilled all four criteria) and $70(40 \%)$ as having "probable" catastrophic APS.

The characteristics of patients classified as having "probable" catastrophic APS were as follows: two patients (3\%) fulfilled all four criteria, except that only two organs, systems, or tissues were involved; nine (13\%) fulfilled all four criteria, except for the absence of laboratory confirmation with at least six weeks owing to the early death of a patient never tested for aPL before the catastrophic APS; and 59 (84\%) fulfilled criteria 1, 2, and 4.

Only one patient from the control group fulfilled criteria for "probable" catastrophic APS. According to these findings, the sensitivity of the preliminary criteria for the classification of catastrophic APS is $90.3 \%$, the specificity $99.4 \%$, the positive predictive value $99.4 \%$, and the negative predictive value $91.1 \%$.

\section{DISCUSSION}

Over the last 10 years, various different case reports and small series have described patients with catastrophic APS. Two major paper summarised the different characteristics of a total of 130 patients and provided information on the pathogenesis, clinical features, treatment, and outcome. ${ }^{89}$ The website based CAPS registry has also compiled a large amount of information but the present study indicates that additional efforts should be made in the future because the registry often receives insufficient data or information on patients who do not have "definite" or "probable" catastrophic APS from physicians all over the world.

Recognition of catastrophic APS has increased over the past years, and it is now clear that it is not only associated with SLE or primary APS, but also with other autoimmune conditions such as rheumatoid arthritis, systemic sclerosis, intestinal inflammatory diseases, and Behçet's disease, among others. Despite earlier recognition and better knowledge of the pathophysiology, the mortality rate is still unacceptably high $(48 \%)$, specially in SLE patients and in patients with cardiac, pulmonary, renal, splenic, and adrenal involvement.

Table 4 Preliminary criteria for the classification of catastrophic antiphospholipid syndrome ${ }^{4}$

(1) Evidence of involvement of three or more organs, systems, and/or tissues*

(2) Development of manifestations simultaneously or in less than one week

(3) Confirmation by histopathology of small vessel occlusion in at least one organ or tissue

(4) Laboratory confirmation of the presence of antiphospholipid antibodies (lupus anticoagulant and/or anticardiolipin antibodies) $\ddagger$

Definite catastrophic APS: all four criteria

Probable catastrophic APS-any of the following:

(a) All four criteria, except for only two organs, systems, and/or tissues involved

(b) All four criteria, except for the absence of laboratory confirmation (within at least 6 weeks) owing to the early death of a patient never tested for aPL before the catastrophic APS

(c) Criteria (1), (2), and (4)

(d) Criteria (1), (3), and (4) and the development of a third event between one week and one month after presentation, despite anticoagulation

*Usually clinical evidence of vessel occlusions, confirmed by imaging techniques when appropriate. Renal involvement is defined by a $50 \%$ rise in serum creatinine, severe systemic hypertension ( $>180 / 100 \mathrm{~mm} \mathrm{Hg}$ ). and/ or proteinuria (>500 mg/24 hours)

†For histopathological confirmation, significant evidence of thrombosis must be present, although vasculitis may coexist occasionally.

\#If the patient had not previously been diagnosed as having an APS, the laboratory confirmation requires that the presence of antiphospholipid antibodies must be detected on two or more occasions at least six weeks apart (not necessarily at the time of the event), according to the proposed preliminary criteria for the classification of definite APS. 
Recently, Erkan et al ${ }^{3}$ evaluated the clinical outcome of 58 survivors of a catastrophic APS event. Thirty eight patients $(66 \%)$ did not develop further APS related events, 15 (26\%) developed a new thrombotic episode (in 13 cases during anticoagulation therapy), but none of them developed further catastrophic APS episodes.

The clinical approach to the treatment of catastrophic APS will depend on the site and extension of the vascular occlusions and the degree of systemic inflammatory response. The cornerstone of the treatment includes readiness to suspect the condition and the treatment of any precipitating factor, especially adequate antibiotic therapy for related infections based on the clinical setting, appropriate anticoagulant management, and the use of immunosuppressive drugs (especially steroids), plus third line therapy (plasma exchange or intravenous immunoglubulins) for the treatment of the thrombotic and cytokine "storm". ${ }^{4}$ Finally, a series of life support measures are needed, such as mechanical ventilation, inotropic drugs, and continuous haemodialysis. ${ }^{10}$

The differential diagnosis in some circumstances is very difficult, specially with other microangiopathic syndromes that are capable of producing multiorgan thrombotic events. These conditions include thrombotic thrombocytopenic purpura, haemolytic-uraemic syndrome, heparin induced thrombocytopenia, and the HELLP (haemolysis, elevated liver enzymes, and low platelet count) syndrome. ${ }^{11}$ In these critically ill patients there is a high chance that their blood samples may show false positive results in lupus anticoagulant assays (for example, coagulation factor deficiencies or heparin use), or that treatment decisions have to be made before the results of laboratory tests are available. However, the presence of persistent positive levels of aPL in a patient with these conditions will lead to the diagnosis of concomitant catastrophic APS. In fact, several patients in the CAPS registry fulfilled criteria for thrombocytopenic purpura or HELLP as well. Thus we only analysed patients with aPL as controls-including both SLE and primary APS patientsand for this reason we did not include controls with multiorgan thrombotic events (for example, thrombotic thrombocytopenic purpura, haemolytic-uraemic syndrome, or HELLP) but without aPL.

Though microthrombosis is one of the typical markers of a catastrophic APS event, it may be difficult to confirm, and many patients could only be labelled as "probable" catastrophic APS based on large vessel multiorgan thrombotic involvement over a short period of time in the presence of aPL. Because of these difficulties in the confirmation of a definite catastrophic APS event, we included both "definite" and "probable" catastrophic APS in the evaluation of the classification criteria. According to our results, the International Consensus Statement on Preliminary Classification Criteria for the catastrophic APS is a useful tool for epidemiological studies and it is hoped that these criteria will be tested in future prospective multicentre studies, and that modifications or additions to the criteria will be made at subsequent workshops. It should be emphasised that these criteria are mostly empirical and have been accepted for classification purposes only. They are not intended to be used as strict diagnostic criteria in a given patient.

\section{ACKNOWLEDGEMENTS}

This study was partially presented at the 2003 Annual European Congress of Rheumatology and was awarded with the European League Against Rheumatism (EULAR)/Abbott Abstract Prize.

\section{Authors' affiliations}

R Cervera, J Font, J A Gómez-Puerta, G Espinosa, M Cucho, S Bucciarelli, M Ramos-Casals, M Ingelmo, Department of Autoimmune Diseases, Institut Clínic de Medicina i Dermatologia, Hospital Clínic, Barcelona, Catalonia, Spain
J-C Piette, Department of Internal Medicine, Hôpital Pitié-Salpêtrière, Paris, France

Y Shoenfeld, Chaim-Sheba Medical Centre, Sackler Faculty of Medicine, Tel-Aviv University, Tel-Hashomer, Israel

R A Asherson, Rheumatic Diseases Unit, University of Cape Town, Faculty of Health Sciences, Cape Town, South Africa

\section{APPENDIX}

\section{THE CATASTROPHIC ANTIPHOSPHOLIPID SYNDROME REGISTRY PROJECT GROUP}

The members of the Catastrophic APS Registry Project Group who contributed with clinical data to this study are as follows: Mary-Carmen Amigo, Rheumatology Department, Instituto Nacional de Cardiología, Ignacio Chavez, Mexico City, Mexico; Leonor Barile-Fabris, Rheumatology Department, Hospital de Especialidades, Centro Medico la Raza IMSS, Mexico City, Mexico; Jean-Jacques Boffa, Department of Nephrology, Hôpital Tenon, Paris, France; Joab Chapman, Neuroimmunology Service, Tel Aviv Sourasky Medical Centre, Tel Aviv, Israel; Christopher Davidson, Department of Cardiology, Royal Sussex Hospital, Brighton, UK; Alex E Denes, Division of Oncology, Department of Medicine, Washington University School of Medicine, St Louis, Missouri, USA; Ronald H W M Derksen, Department of Rheumatology and Clinical Immunology, University Medical Centre, Utrecht, Netherlands; J F Diaz Coto, Caja Costarricense del Seguro Social, San Jose, Costa Rica; Patrick Disdier, Service de Medecine Interne, Centre Hospitalier Universitaire Timone, Marseille, France; Rita M Egan, Department of Medicine, University of Kentucky Medical Center, Lexington, Kentucky, USA; M Ehrenfeld, Chaim Sheba Medical Centre and Tel-Aviv University, Tel-Hashomer, Israel; R Enriquez, Nephrology Section, Hospital General de Elche, Spain; Doruk Erkan, Hospital for Special Surgery, New York, USA; Fernanfa Falcini, Department of Paediatrics, University of Florence, Italy; Leslie S Fang, Renal Associates, Massachusetts General Hospital and Harvard Medical School, Boston, Massachusetts, USA; Mario García-Carrasco, Benemérita Universidad Autónoma de Puebla, Puebla, Mexico; John T Grandone, Neenah, Wisconsin, USA; Anagha Gurjal, Division of Hematology/Oncology, Barbara Ann Karmanos Cancer Institute, Detroit, Michigan, USA; Gilles Hayem, Department of Rheumatology, CHU Bichat-ClaudeBernard, Paris, France; Graham R V Hughes, Lupus Research Unit, The Rayne Institute, St Thomas' Hospital, London, UK; Sohail Inam, Riyadh Armed Forces Hospital Riyadh, Saudi Arabia; K Shashi Kant, Department of Internal Medicine, University of Cincinnati College of Medicine, Ohio, USA; Munther A Khamashta, Lupus Research Unit, The Rayne Institute, St Thomas' Hospital, London, UK; Craig S Kitchens, Department of Medicine, University of Florida, Gainesville, Florida, USA; Michael J Kupferminc, Department of Obstetrics and Gynaecology, Lis Maternity Hospital, Tel Aviv University, Tel Aviv, Israel; Gabriela de Larrañaga, Hospital Muñiz, Buenos Aires, Argentina; Roger A Levy, Department of Rheumatology, Faculdade de Ciencias Medicas, Universidade do Estado do Rio de Janeiro, Rio de Janeiro, Brazil; Daryl Tan, Singapore General Hospital, Singapore; Siu Fai Lui, Department of Medicine, Prince of Wales Hospital and Chinese University of Hong Kong, Shatin, Hong Kong; Peter J Maddison, Gwynedd Rheumatology Service, Ysbyty Gwynedd, Bangor, UK; Yoseph A Mekori, Department of Medicine, Meir Hospital, Kfar Saba, Israel; Takako Miyamae, Department of Paediatrics, Yokohama City University School of Medicine, Yokohama, Japan; John Moore, Department of Haematology, St Vincents Hospital, Sydney, Australia; Haralampos M Moutsopoulos, Department of Pathophysiology, Medical School, National University of Athens, Athens, Greece; Francisco J Munoz-Rodriguez, Department of Autoimmune Diseases, Hospital Clinic, Barcelona, Catalonia, Spain; Jacek Musial, Jagiellonian University School of Medicine, Krakow, Poland; Ayako Nakajima, Institute of Rheumatology, Tokyo Women's Medical University, Tokyo, Japan; Michael C Neuwelt, Medical Service, VA Palo Alto Health Care System, California, USA; 
Ann Parke, Department of Internal Medicine, Division of Rheumatic Diseases, University of Connecticut Health Center, Connecticut, USA; Sonja Praprotnik, University Clinical Centre, Department of Rheumatology, Ljubljana, Slovenia; Bernardino Roca, Department of Internal Medicine, Hospital General de Castelló, Castelló, Spain; Jorge Rojas-Rodriguez, Department of Rheumatology, Specialties Hospital, Manuel Avila Camacho National Medical Centre, Puebla, Mexico; R Roldan, Rheumatology Department, Hospital Reina Sofia, Cordoba, Spain; Allen D Sawitzke, Division of Rheumatology, Department of Internal Medicine, University of Utah School of Medicine, Salt Lake City, Utah, USA; Cees G Schaar, Department of Haematology, Leiden University Medical Centre, The Netherlands; Alenka Šipek-Dolnicar, Department of Rheumatology, University Medical Centre, Ljubljana, Slovenia; Alex C Spyropoulos, Clinical Thrombosis Center, Albuquerque, New Mexico, USA; Renato Sinico, Nephrology and Dialysis Unit and Centre of Clinical Immunology and Rheumatology, San Carlo Borromeo Hospital, Milan, Italy; Ljudmila Stojanovich, Clinical-Hospital Centre "Bezhanijska Kosa", Belgrade, Yugoslavia; Marcos Oaulo Veloso, Hospital Universitario Clementino Fraga Filho, Rio de Janeiro, Brazil; Maria Tektonidou, Department of Pathophysiology, Medical School, National University of Athens, Athens, Greece; Carlos Vasconcelos, Hospital General de San Antonio, Porto, Portugal; Marcos Paulo Veloso, Hospital Universitario Clementino Fraga Filho, Rio de Janeiro, Brazil; Margaret Wislowska, Outpatients Department of Rheumatology, Central Clinical Hospital, Warsaw, Poland.

\section{REFERENCES}

1 Asherson RA. The catastrophic antiphospholipid syndrome. J Rheumatol 1992; 19:508-12.

2 Cervera R, Piette JC, Font J, Khamashta MA, Shoenfeld Y, Camps MT, et al. Antiphospholipid syndrome: clinical and immunologic manifestations and patterns of disease expression in a cohort of 1000 patients. Arthritis Rheum 2002;46:1019-27.

3 Erkan D, Asherson RA, Espinosa G, Cervera R, Font J, Piette JC, et al. Long term outcome of catastrophic antiphospholipid syndrome survivors. Ann Rheum Dis 2003;62:530-3.

4 Asherson RA, Cervera R, de Groot PG, Erkan D, Boffa MC, Piette JC, et al. Catastrophic antiphospholipid syndrome: international consensus statement on classification criteria and treatment guidelines. Lupus 2003; 12:530-4.

5 Tan EM, Cohen AS, Fries JF, Masi AT, McShane DJ, Rothfield NF, et al. The 1982 revised criteria for the classification of systemic lupus erythematosus. Arthritis Rheum 1982;25:1271-7.

6 Wilson WA, Gharavi AE, Koike T, Lockshin MD, Branch DW, Piette JC, et al. International consensus statement on preliminary classification criteria for definite antiphospholipid syndrome: report of an international workshop. Arthritis Rheum 1999;42:1309-11.

7 Galen RS, Gambino RS. Beyond normality: the predictive value and efficiency of medical diagnoses. New York: John Wiley, 1975.

8 Asherson RA, Cervera R, Piette JC, Font J, Lie JT, Burcoglu A, et al. Catastrophic antiphospholipid syndrome. Clinical and laboratory features of 50 patients. Medicine (Baltimore) 1998;77:195-207.

9 Asherson RA, Cervera R, Piette JC, Shoenfeld Y, Espinosa G, Petri MA, et al. Catastrophic antiphospholipid syndrome: clues to the pathogenesis from a series of 80 patients. Medicine (Baltimore) 2001;80:355-77.

10 Westney GE, Harris EN. Catastrophic antiphospholipid syndrome in the intensive care unit. Crit Care Clin 2002;18:805-17.

11 Asherson RA, Cervera R. Catastrophic antiphospholipid syndrome. Curr Opin Hematol 2000;7:325-9. 